\title{
Charge carrier generation in photosensitive amorphous molecular semiconductors
}

\author{
M.A. Zabolotny \\ T. Shevchenko Kyiv National University, 64 vul. Volodymirska, 01033 Kyiv, Ukraine
}

\begin{abstract}
Thermalization process in photosensitive amorphous molecular semiconductors are theoretically considered from the standpoint of their parameters, namely: thermalization time, thermalization length. The heat electron formed in consequence of absorption of the light quantum by semiconductor molecules loses his surplus energy in the course of inelastic interaction with neighbouring atoms. The results of theoretical predictions are confirmed by the experimental ones obtained for a number of molecular semiconductors (anthracene, pentacene, PVC, PEPC).
\end{abstract}

Keywords: amorphous molecular semiconductors, thermalization time, thermalization length.

Paper received 15.01.03; accepted for publication 18.03.03.

\section{Introduction}

Amorphous molecular semiconductors (AMS) based on sensibilized photoconducting polymers possess photoconducting properties in the visual and near IR wavelength range [1]. The reason for this is that sensibilizer molecules act not only as centers of light absorption but also as centers of charge carriers photogeneration. These properties of AMS make it possible to utilize them for development of microelectronic systems [1]. The best-studied and widely used AMS are those based on poly-N-vinylecarbazole (PVC), poly-N-epoxypropylcarbazole (PEPC).

It is known [2] that for this type of AMS the photogeneration process consists of several steps. However, there are two main stages. The first one takes about $10^{-11}$ $10^{-12} \mathrm{~s}[3,4]$; during this stage, after absorption of the light quantum, the neutral exciton state arises and due to autoionization transforms into an ion-"hot" charge carrier pair. The latter releases excessive energy via inelastic interaction with surrounding atoms and during the thermalization time $t_{\tau}$ moves to the thermalization length $r_{\tau}$.

The thermalization process is considered to be finished when the excessive energy of the non-equilibrium charge carries has decreased to such a value DW that its interaction with surrounding becomes elastic. The thermalization leads to the transfer of the mobile charge carrier from the photogeneration center to the carbazole nucleus of AMS that in turn leads to electron-hole pair formation. Then the second stage begins. During this stage, dissociation of the electron-hole pair occurs at thermal equilibrium with surrounding. This stage can be satisfactory described by Onsager model, or its generalizations [5] that take into account a possibility of tunneling processes. There is, however, no adequate model for the first stage of the process of charge generation in AMSs. The subject of this work is to develop a model for the thermalization of the charge carriers in AMS.

The most useful information to be gained from the experimental studies of different stages of the photogeneration of charge carriers is the quantum yield dependence on the wavelength of exciting light, because it is determined only by the thermalization process. The photogeneration quantum yield $\eta$ dependence in anthracene and pentacene [2, 4], as well as in PVC, PEPC, were studied in $[6,7]$. It was found that the thermalization length increases with the frequency of the light. These results are presented in Figs 1, 2. 
M.A. Zabolotny: Charge carrier generation in photosensitive ...

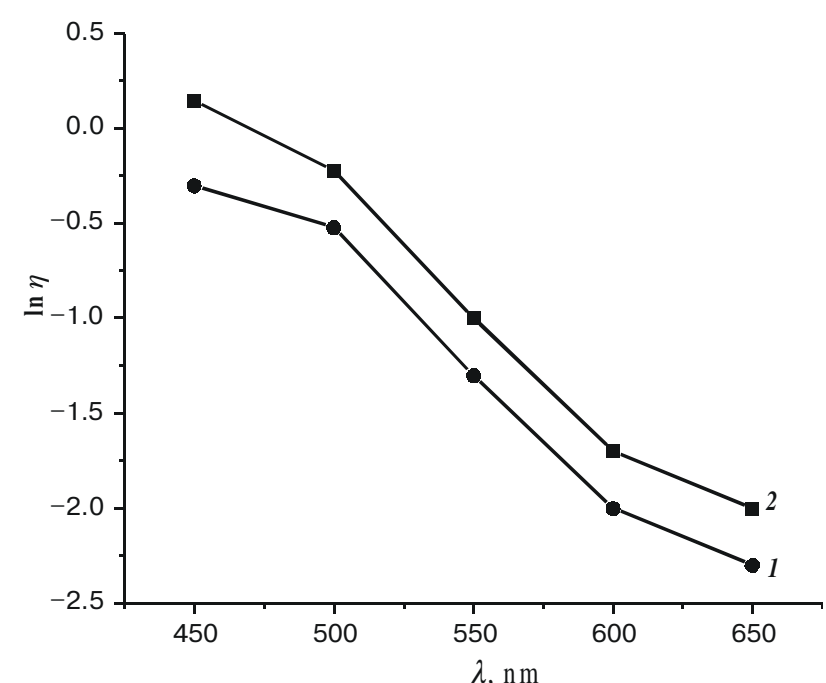

Fig. 1. The experimental dependence of quantum yield of photogeneration on wave length light (curve 1 - PVC, 5 mol. \% TNF), (curve 2 - PVC-Luvican, 5 mol. \% TNF).

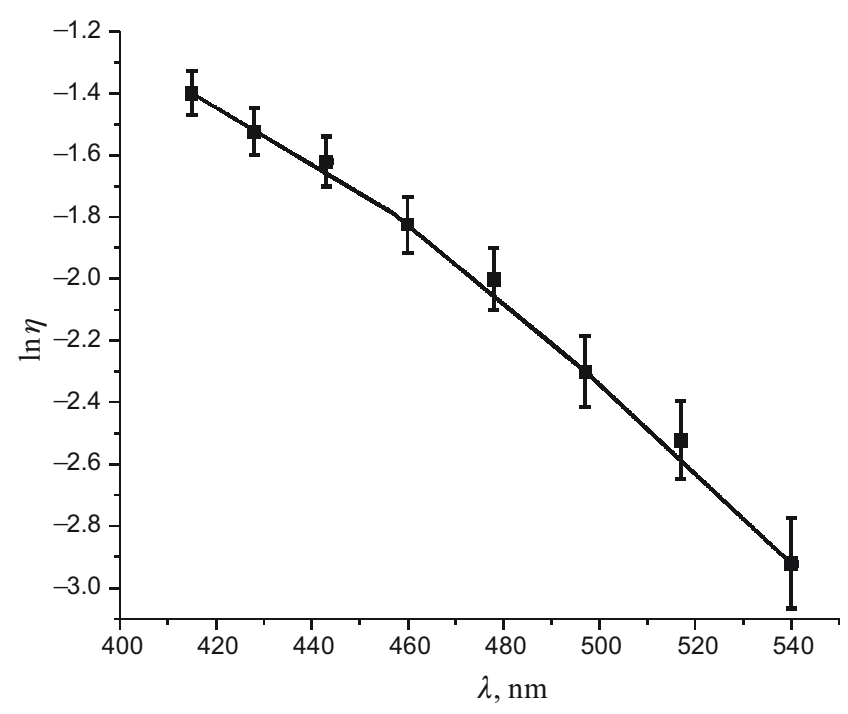

Fig. 2. The experimental dependence of quantum yield of photogeneration in pentacene on the light wave length.

\section{Thermalization process model}

The model, based on the assumption that the energy excess of the non-equilibrium charge carrier decreases due to it transfers to the surrounding molecules, is used for explanation of the quantum yield dependence on the exciting wavelength. The potential energy is taken to be constant; AMS molecules do not change their position and structure when the non-equilibrium charge carrier is passing by.

In such an approach, excessive energy of the nonequilibrium charge carrier is determined by its kinetic energy $E(t)$. This allows to relate the non-equilibrium charge carrier velocity $v(t)$ and its temperature $T(t)$ : $v(t)=\sqrt{\frac{3 k_{b} T(t)}{m}}$.

Here, $k_{b}$ is the Boltzmann constant and $m$ is the mass of the non-equilibrium charge carrier.

Let's take into account that the non-equilibrium carriers ensemble becomes quasi-equilibrium with temperature $\left(\Delta T+T_{0}\right)$ when the excessive energy achieves a value $\Delta W$; here $T_{0}$ is the temperature of surrounding. Using the fact that the motion of the charge carrier is rectilinear during thermalization, the thermalization length can be defined as:

$r_{\tau}=\int_{0}^{t_{c}} v(t) d t=\sqrt{\frac{3 k_{b}}{m}} \cdot \int_{0}^{t_{c}} \sqrt{T(t)} d t$

It is assumed that heat transfer process is Newtonian, i.e. the heat transfer rate is proportional to the temperature difference $\left(T(t)-T_{0}\right)$. Then, the temperature kinetics of the non-equilibrium charge carrier is described by the following equation:

$\frac{d T(t)}{d t}=-\frac{2 \chi}{3 k_{b}}\left(T(t)-T_{0}\right)$

Here, $\chi$ is the parameter that determines the heat transfer rate. As an initial condition it is used that $T(0)$ is determined by the energy $\hbar \omega$ of the absorbed light quantum and the work $A$ that is required in order to create exciton state [2]

$T(0)=-\frac{2}{3 k_{b}}(\hbar \omega-A)$.

Hence,

$T(t)=T_{0}\left(1-\exp \left(-\frac{2 \chi}{3 k_{b}} t\right)\right)+$

$+\frac{2}{3 k_{b}}(\hbar \omega-A) \exp \left(-\frac{2 \chi}{3 k_{b}} t\right)$

Using equation (5) and the following condition:

$T\left(t_{\tau}\right)=T_{0}-\Delta T$

an expression for $t_{\tau}$ is obtained:

$t_{\tau}=\frac{3 k_{b}}{2 \chi} \ln \left[\frac{1}{\Delta T}\left(\frac{2(\hbar \omega-A)}{3 k_{b}}\right)-T_{0}\right]$.

It follows from (2) and (7) that

$r_{\tau}=\sqrt{\frac{2}{m}(\hbar \omega-A)} \cdot \frac{3 k_{b}}{\chi} \cdot\left[1-\sqrt{\frac{2}{3 k_{b}}(\hbar \omega-A)-T_{0}}\right]$ 


\section{M.A. Zabolotny: Charge carrier generation in photosensitive ...}

Expressions (7) and (8) allow to find relation between $t_{\tau}$ and $r_{\tau}$ that is independent on the unknown parameter $\chi$ :

$$
\begin{aligned}
& r_{\tau}=\frac{2 \sqrt{\frac{2}{m}(\hbar \omega-A)}}{\ln \left(\frac{1}{\Delta T}\left(\frac{2(\hbar \omega-A)}{3 k_{b}}-T_{0}\right)\right)} \times \\
& \times\left[1-\sqrt{\frac{2}{3 k_{b}}(\hbar \omega-A)-T_{0}}\right] t_{\tau}
\end{aligned}
$$

\section{Discussion of results and conclusions}

It follows from (8) that $r_{\tau}$ increases with $\hbar \omega$; this is in good agreement with the data summarized in Table as well as those published earlier [1,4]. It should be noted that dependence $r_{\tau}(\omega)$ correlated well with results of the model that assumes lost of excessive energy of non-equilibrium charge carrier due to collisions with surrounding molecules at rest [8]. It also follows from the expression (8) that thermalisation length increases as surrounding temperature decreases, which has been observed experimentally [2,4]. Substituting $t_{\tau} \sim 10^{-12} \mathrm{~s}$ into the relation (9) allows to estimate $r_{\tau}$ giving $r_{\tau} \sim\left(10^{-9}-10^{-8}\right) \mathrm{m}$ that agrees with experimental results $[2,6]$. Therefore, the proposed model of thermalization process of the charge carriers explains qualitatively the spectral dependencies of the quantum yield of photogeneration and gives the relation between $r_{\tau}$ and $t_{\tau}$ that is in a good agreement with experimental results.

\section{References}

1. L. Fabbrizi, A.Poggi, Sensor and Switches from Supramolecular Chemistry // Chem. Soc. Review, p. 197-202 (1995).

2. E.A. Silinsh, M.V. Kurik, V. Capek, Electronic processes in organic molecular crystals // Riga, Zinatne, p.329 (1988) (in Russian).

3. L. Valkunas, V. Gubinas, A. Undzenas, Charge separation and recombination in polymers and aggregates // Proc. Of Int Conf. Electronic Processes in Organic Materials 22B, p. 54 (1998)

4. E.A. Silinsh, V.A. Kollesnikov, I.J. Muzikante, D.R. Balode, On charge carriers photogeneration mechanisms in organic molecular crystals // Phys. Status Solidi (b) 113, p. $379-$ 393 (1982).

5. M.A. Zabolotny, A.G. Zagorodny, N.P. Borolina, Influence of tunneling on formation of charge carriers in polymeric semiconductors in electrical field // Phys. Status Solidi 33(8), p. 2345-2349 (1991) (in Russian).

6. E.L. Alexandrova, The spectral dependence of the quantum yield in complexes with a charge transfer PVC TNDZM // Proc. of conf. The silverlos and unusual photographic processes 1, part 1, Chernogolovka, p. 95-96 (1984) (in Russian).

7. Yu.M. Barabash, M.A. Zabolotny, N.I. Sokolov, Photogeneration of charge carriers in photosensitive organic semiconductors // Semiconductors physics, quantum electronics and optoelectronics 2(4), p. 51-54 (1999).

8. A.I. Nazarov, V.V. Sergeeva, Calculation of quantum yield of nonequilibrium charge carriers in a dielectric under ionizing irradiation // Journal Techn. Physics 67(6), p. 127-130 (1997) (in Russian). 